\title{
Time-dependent spectral-feature variations of stars displaying the $B[e]$ phenomenon
}

\author{
I. V2028 Cygni ${ }^{\star, \star \star}$ \\ J. Polster ${ }^{1,2}$, D. Korčáková ${ }^{2}$, V. Votruba ${ }^{1,3}$, P. Škoda ${ }^{3}$, M. Šlechta ${ }^{3}$, B. Kučerová ${ }^{1}$, and J. Kubát ${ }^{3}$ \\ ${ }^{1}$ Faculty of Science, Masaryk University in Brno, Kotlářská 2, 61137 Brno, Czech Republic \\ e-mail: jpolster@email.cz \\ 2 Astronomical Institute, Charles University in Prague, V Holešovičkách 2, 18000 Praha 8, Czech Republic \\ e-mail: kor@sirrah.troja.mff.cuni.cz \\ 3 Astronomical Institute of the Academy of Sciences of the Czech Republic, Fričova 298, 25165 Ondřejov, Czech Republic
}

Received 7 May 2011 / Accepted 27 March 2012

\section{ABSTRACT}

\begin{abstract}
We present results of nearly six years of spectroscopic observations of the B[e] star V2028 Cyg. The presence of the cold-type absorption lines combined with a hot-type spectrum indicate the binarity of this object. Since B[e] stars are embedded in an extended envelope, the usage of common stellar atmosphere models for the analysis is quite inappropriate. Therefore, we focus on the analysis of the long-term spectral line variations in order to determine the nature of this object. We present the time dependences of the equivalent width and radial velocities of the $\mathrm{H} \alpha$ line, [O I] $6300 \AA$, Fe II 6427, 6433, and $6456 \AA$ lines. The bisector variations and line intensities are shown for the $\mathrm{H} \alpha$ line. The radial velocities are also measured for the absorption lines of the K component. No periodic variation is found. The observed data show correlations between the measured quantities, which can be used in future modelling.
\end{abstract}

Key words. line: profiles - binaries: general - circumstellar matter - stars: emission-line, Be - stars: individual

\section{Introduction}

V2028 Cyg (MWC 623) belongs to a rather heterogeneous group called $\mathrm{B}[\mathrm{e}]$ stars. This group includes such dissimilar objects as compact planetary nebulae, Herbig stars, and supergiants or symbiotic stars (Lamers et al. 1998). A large number of $\mathrm{B}[\mathrm{e}]$ stars still belong to this "unclassified" subgroup, which is also the case for V2028 Cyg. It is difficult to assign this object to a specific subgroup because of the presence of a circumstellar matter. Miroshnichenko (2007) found that many unclassified objects display common spectral signatures. He denoted this group as FS CMa stars, since this star can be taken as a good prototype for these objects. He included in this group V2028 Cyg. Current observation analysis shows that they are nonsupergiants. FS CMa objects are probably more evolved stars, but are either close to the main sequence, or still on it (Miroshnichenko et al. 2000; Miroshnichenko 2007). Miroshnichenko (2007) suggested that a binarity could explain the observed properties of these stars.

The commonly used model of $\mathrm{B}[\mathrm{e}]$ stars was proposed by Zickgraf et al. (1985). The B[e] phenomenon arises in a twocomponent stellar wind with a dusty ring. A slow, cool, high density wind occurs around the equatorial plane. This wind forms an outflowing disc, where the emissions of both the Balmer series and low-ionized metals originate. The polar areas are occupied by a CAK (Castor et al. 1975) wind

$\star$ Based on data from the Ondřejov $2 \mathrm{~m}$ telescope, Czech Republic. $\star \star$ Reduced Ondrejov spectra are only available at the CDS via anonymous ftp to cdsarc.u-strasbg.fr $(130.79 .128 .5)$ or via http://cdsarc.u-strasbg.fr/viz-bin/qcat?J/A+A/542/A57 of a low density and high velocity. The gas in this outflow is hot and the emission lines come from metals in higher ionization stages.

V2028 Cyg was first noted as an emission-line star by Merrill et al. (1942). Allen \& Swings (1976) discovered its photometrical variability (amplitude $0.5 \mathrm{mag}$ in filter $V$ ). Bergner et al. (1995) then presented the amplitude 0.2-0.4 mag. Allen (1973) analysed photometric measurements from the filters $H$, $K$, and $L$ and reported an infrared excess that he explained in terms of a dust envelope. Allen (1974) then noted the connection between the excess and forbidden emission lines. A few years later, Allen \& Swings (1976) published a spectral analysis of the group of peculiar Be stars with infrared excesses, including V2028 Cyg. They identified permitted and forbidden emission lines, such as Balmer lines, Fe II, [OII, and [Fe II], which dominate the emission spectrum.

V2028 Cyg was noted for the first time as a binary by Arkhipova \& Ipatov (1982). They suggested spectral the types B8 and M1III for the system from the analysis of spectrophotometric observations. However, they used assumptions that were later found to be incorrect. High resolution spectra enabled Zickgraf \& Stahl (1989) to identify many absorption lines of mainly neutral metals, such as Fe I, Ti I, VI, CaI, and Li I (also detected later by Corporon \& Lagrange 1999; and Zickgraf 2001). By fitting theoretical spectral energy distribution to photometric data, they suggested that V2028 Cyg is a binary composed of a B2 dwarf and a K2 giant. From comparison with spectra of normal stars, Zickgraf (2001) refined the component classification to B4III and K2Ib-II. Multicolour optical and near-infrared photometric 


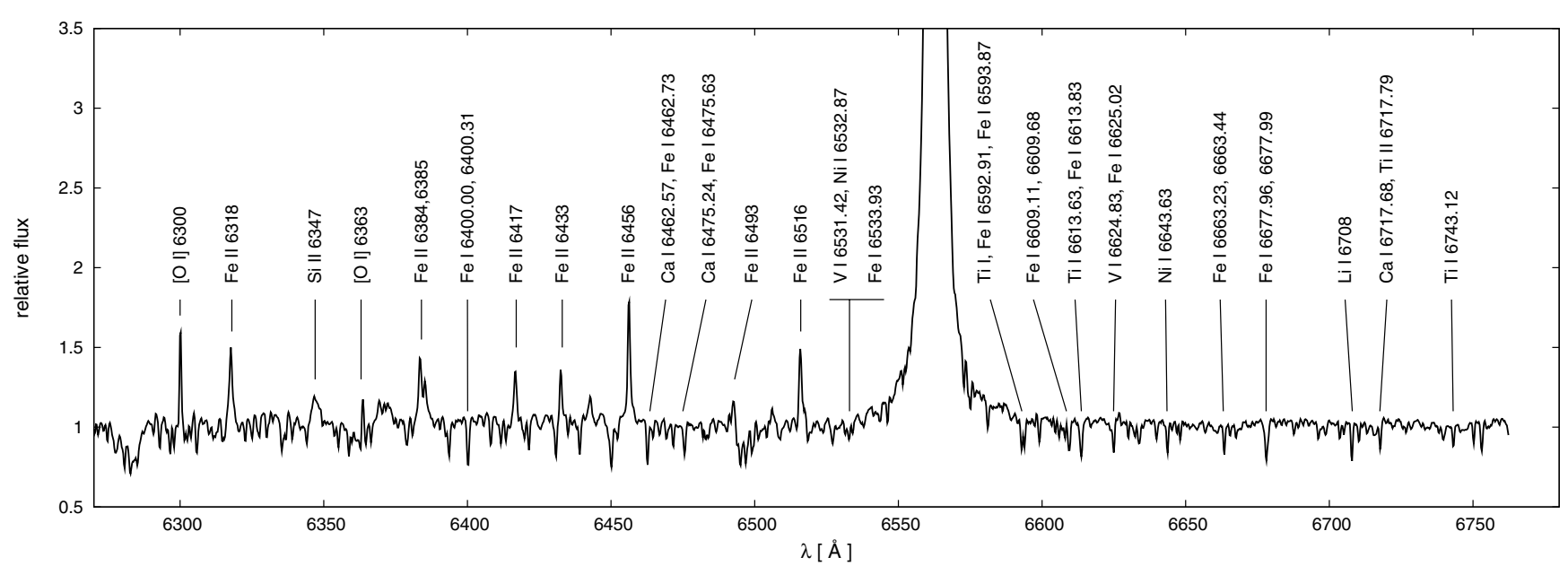

Fig. 1. Spectral interval around $\mathrm{H} \alpha$ observed at the Ondřejov observatory. $\mathrm{H} \alpha$ profile is cut to resolve other spectral lines.

observations led Bergner et al. (1995) to derive the type K7III for the cool component. The primary component falls on the HR diagram into the region where other FS CMa stars are also found (Miroshnichenko 2007). Its position close to the region occupied by classical Be stars, provides a good opportunity to study the connection between the Be and $\mathrm{B}[\mathrm{e}]$ phenomenon.

Zickgraf (2001) derived a kinematical distance towards V2028 Cyg using the average radial velocity $\left(2.0_{-0.3}^{+0.6} \mathrm{kpc}\right)$ and his luminosity estimate for both components $\left(2.4_{-0.9}^{+1.4} \mathrm{kpc}\right)$.

The optical emission spectrum consists of a large number of singly ionized metallic lines (Fe II, Ti II, $\mathrm{Cr}$ II, $\mathrm{Si}$ II) and is dominated by the hydrogen lines of the Balmer series. V2028 Cyg possesses an extended gaseous and dusty envelope, which is indicated by several spectral features (IR excess, forbidden lines, intensive Balmer emission). Marston \& McCollum (2008) unsuccessfully tried to find evidence of larger-scale structures (lobes, nebula, ...) around the object using a narrow-band $\mathrm{H} \alpha$ filter.

Zickgraf \& Schulte-Ladbeck (1989) assumed that a circumstellar disc structure causes a direction-dependent polarisation of the emitted photons. They determined an intrinsic polarization of $\sim 2 \%$ from their observations. On the basis of this value and the assumption that the scattering plane is parallel to the orbital plane, Zickgraf (2001) estimated an inclination angle $\geq 30-45^{\circ}$.

The gaseous and dusty envelope of V2028 Cyg makes it very difficult to determine the fundamental parameters of the object, since the commonly used stellar atmosphere models are not applicable in this case. The properties of these objects can only be described by multidimensional models that combine timedependent hydrodynamics and NLTE radiative transfer. This task is our ultimate goal. Our time-dependent hydrodynamical code (Votruba et al. 2007), which is now extended into 2D, gives the temperature and density distributions and velocity of the material for the 2D radiative transfer code (Korčáková \& Kubát 2005; Korčáková et al. 2011). However, this is a longterm project complicated the role of different physical effects in the formation of the $\mathrm{B}[\mathrm{e}]$ phenomenon remaining unclear. Therefore, we decided to focus our efforts here on the analysis of the object's temporal behaviour that may reveal important physical mechanisms and narrow down the possible system configurations. The first papers of the planned series focus on a description of the observed spectral properties of selected $\mathrm{B}[\mathrm{e}]$ stars over a time interval of several years.
The observations, data reduction, and main spectral features are described in Sects. 2 and 3. The following Sect. 4 deals with the temporal variability of the emissions of Fe II (6318, 6456, $6417 \AA$ ), [O I] (6300 ̊), and H $\alpha$. In Sect. 5, we discuss the possible nature of V2028 Cyg based on our observational results and give several criteria and suggestions for future modelling.

\section{Observations and data reduction}

The principal part of our data were obtained with the $2 \mathrm{~m}$ telescope located at the Ondřejov observatory, Czech Republic. We collected 88 spectra around the $\mathrm{H} \alpha$ line during 45 nights over nearly six years (December 1, 2004-October 8, 2010). The wavelength interval in these spectra covers $6250-6770 \AA$ (Fig. 1). The resolving power is $R \sim 12500$. Three additional spectral intervals were observed: 7520-8030 $\AA$ (seven spectra), 7700-8210 ̊ (three spectra), and 8200-8700 (three spectra). The Ondřejov spectra were taken with the classical spectrograph at the Coudé focus (Škoda et al. 2002).

This data set is supplemented by four spectra (acquired over the years 1994-1996) obtained with an èchelle spectrograph ELODIE operated at the Cassegrain focus of the $1.93 \mathrm{~m}$ telescope at the Observatoire de Haute-Provence. The resolving power is 42000 (Moultaka et al. 2004) in the wavelength interval 4000-6800 A.

Data reduction was performed using the $\mathrm{IRAF}^{1}$ data reduction package, and the DCR program (Pych 2004) was used for cosmic-ray-hit elimination.

\section{Spectral features}

As mentioned above, the spectrum of V2028 Cyg contains both emission and absorption lines. The absorption spectrum is dominated by the lines of the later component. The earlier component absorptions are observed in He I lines (4026, 4144, $4472 \AA$ ). The emission spectrum is dominated by Balmer lines $(\mathrm{H} \alpha-\mathrm{H} \delta)$, which are about an order of magnitude stronger then the remaining emission lines in the spectrum. Most of the emission

\footnotetext{
1 IRAF is distributed by the National Optical Astronomy Observatories, operated by the Association of Universities for Research in Astronomy, Inc., under contract to the National Science Foundation of the United States.
} 

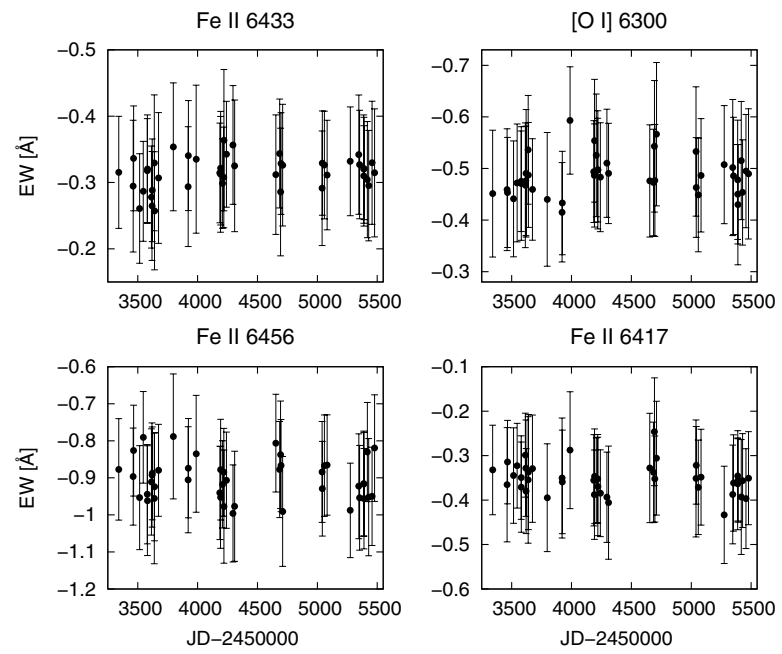

Fig. 2. Equivalent widths of the Fe II and [O I] emission lines.

lines come from singly ionized metals (Fe II, Ti II, Cr II, Si II). Forbidden lines are represented by [Fe II] (4244, 4277, 4287, $7711 \AA$ ) and [OI] $(6300,6364 \AA)$. The emission-line identification is mostly taken from Zickgraf \& Stahl (1989).

\subsection{Equivalent widths}

The equivalent widths of the lines were calculated by a numerical integration of the line profile using a trapezoidal rule. The method of Vollmann \& Eversberg (2006) is adopted for the error estimation. The signal-to-noise ratio $(\mathrm{S} / \mathrm{N})$ of individual spectra was calculated by performing a linear fitting of the continuum around the studied line. The weighting function for the fit was taken from Mikulášek et al. (2003).

The $\mathrm{S} / \mathrm{N}$ around the $\mathrm{H} \alpha$ is about $25-45$. Higher $\mathrm{S} / \mathrm{N}$ values cannot be achieved using the instrument that we used, as the maximum intensity of the $\mathrm{H} \alpha$ line is much stronger than that in the continuum. To obtain an unsaturated $\mathrm{H} \alpha$ line, the signal in the continuum cannot be high (300-900 ADU). The average spectrum was used when more then one observation was made during the night.

The equivalent width measurements of the metallic lines are shown in Fig. 2. The mean values are $-0.31 \pm 0.02 \AA$ (Fe II $6433 \AA),-0.90 \pm 0.05 \AA\left(F e_{\text {II }} 6456 \AA\right),-0.35 \pm 0.03 \AA$ (Fe II $6417 \AA$ ), and $-0.49 \pm 0.04 \AA$ ([O I] $6300 \AA$ ). The relative errors of $10-26 \%$ do not, however, allow us to make a definite statement about the magnitude or periodicity of the variations.

In the case of the $\mathrm{H} \alpha$ line, the equivalent width variations are clearly visible. The relative errors are around $4 \%$. Figure 3 shows the changes in the $\mathrm{H} \alpha$ equivalent width and height. Both quantities exhibit very similar behaviours. Only data from the Ondřejov spectra are plotted in Fig. 3, therefore direct comparison is possible.

\subsection{Line profiles}

The metallic emission lines in the Ondřejov spectra are only slightly asymmetric, and their profiles are very close to a Gaussian. A higher resolution spectra from the ELODIE spectrograph clearly show the asymmetry of metallic emission lines (see Fig. 4).

The blue side of the profile decreases more steeply then the red side, which is evidence of a mass outflow. This feature is
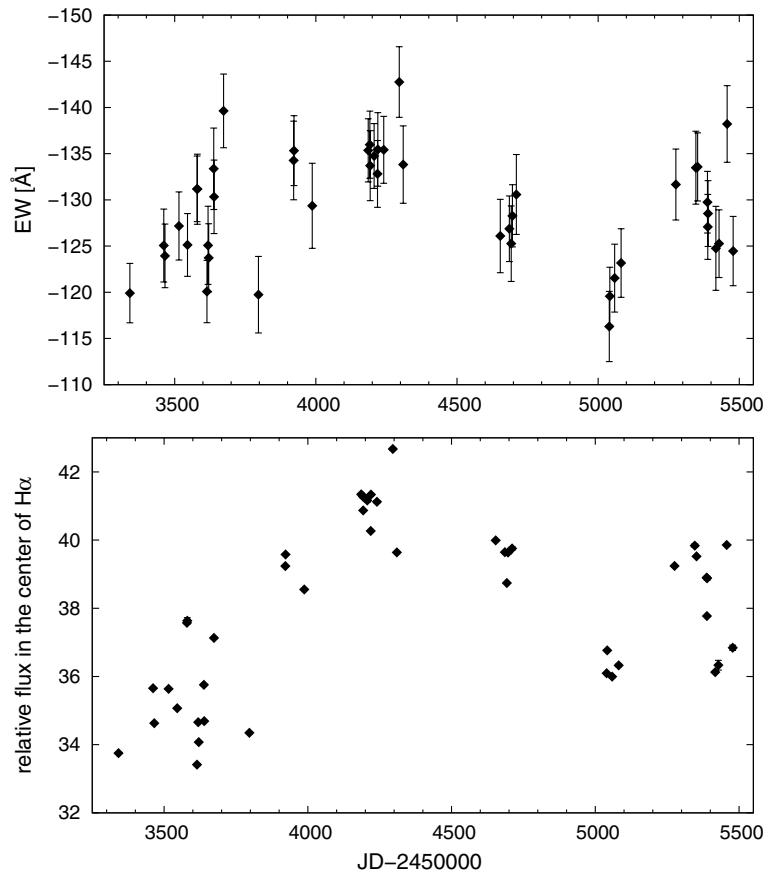

Fig. 3. Equivalent widths (squares) and intensities (diamonds) of the $\mathrm{H} \alpha$ line. Only Ondřejov data are presented. The error bars of intensities are of the same size as the points.
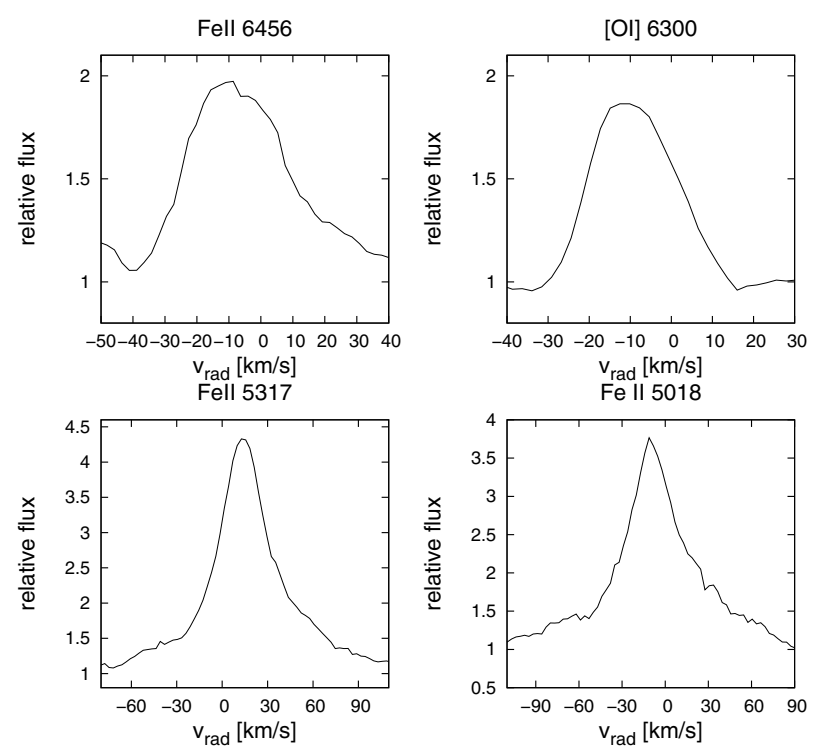

Fig. 4. Emission line profiles of $\mathrm{Fe}$ II 6456, 5317, 5018, and [O I] 6300. ELODIE spectrograph (night August 11/12, 1995).

common in all metallic emission lines stronger than $\sim 1.8$ times the continuum level. The line profile shape of the weaker lines cannot be precisely determined owing to the noise, but in some cases the same type of asymmetry is discernible, and also in the case of the [O I lines. This feature was mentioned before by Zickgraf \& Stahl (1989).

Balmer lines are strongly asymmetric and their asymmetry is somewhat the opposite of that of the metallic lines (Fig. 5). The narrow peak tends to be shifted to the red side with respect to the lower parts of the core. The shift varies in time. The little depressions on the red side of the $\mathrm{H} \alpha$ represent the absorption of atmospheric water. 

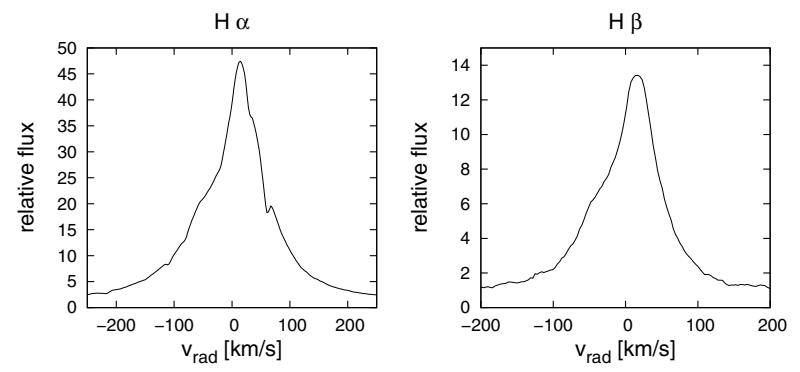

Fig. 5. Emission line profiles of $\mathrm{H} \alpha$ and $\mathrm{H} \beta$. ELODIE spectrograph (night August 11/12, 1995). The line profiles are affected by the telluric lines in this figure.

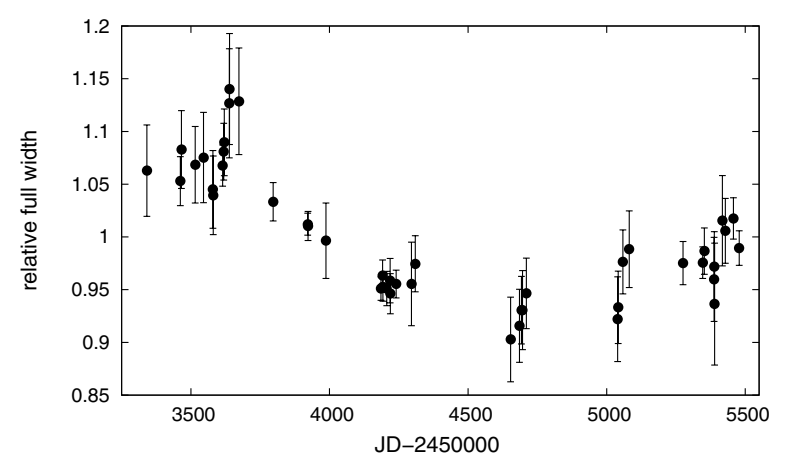

Fig. 6. Variations in the full width of the $\mathrm{H} \alpha$ line. The mean value of the full widths measured in relative heights $0.1-0.9$ is plotted in the graph.

The $\mathrm{H} \alpha$ line is the most pronounced line in the spectrum and its profile is strongly variable. The changes have a noticeable maximum and minimum and are seen not only in the line intensity and equivalent width (Fig. 3), but also in the bisector velocities. Previous observations of Zickgraf (2001) show even stronger variations then those present in our data set. He found that the intensity of the $\mathrm{H} \alpha$ line changed significantly over the years 1986-2000.

\subsubsection{Width and height of $\mathrm{H} \alpha$}

The heights (the maximum flux I; Fig. 3) and widths (Fig. 6) of the $\mathrm{H} \alpha$ line vary during the observation run. The relative differences of the widths are as high as $24 \%$ and for the heights it is $21 \%$.

The absolute value of the equivalent widths $|E W|$ is correlated with height (correlation coefficient $\varrho=0.75$ ), but there is no correlation with the measurements of our so-called full widths $(\varrho=-0.06)$, which were measured at relative heights of from 0.1 (wings) to 0.9 (peak) with a step size of 0.05 . To compare variations in the different relative heights, we divided each of them by its mean value. The relative variations in the individual heights were found to be very similar.

\subsection{2. $\mathrm{H} \alpha$ bisectors}

A more detailed description of the profile variations was done using bisectors. Certain values of the bisectors were measured at the relative heights $0.1-0.9$ with a step of 0.05 . We used the interpolation method of Steffen (1990) to obtain line profile points from the observed ones.
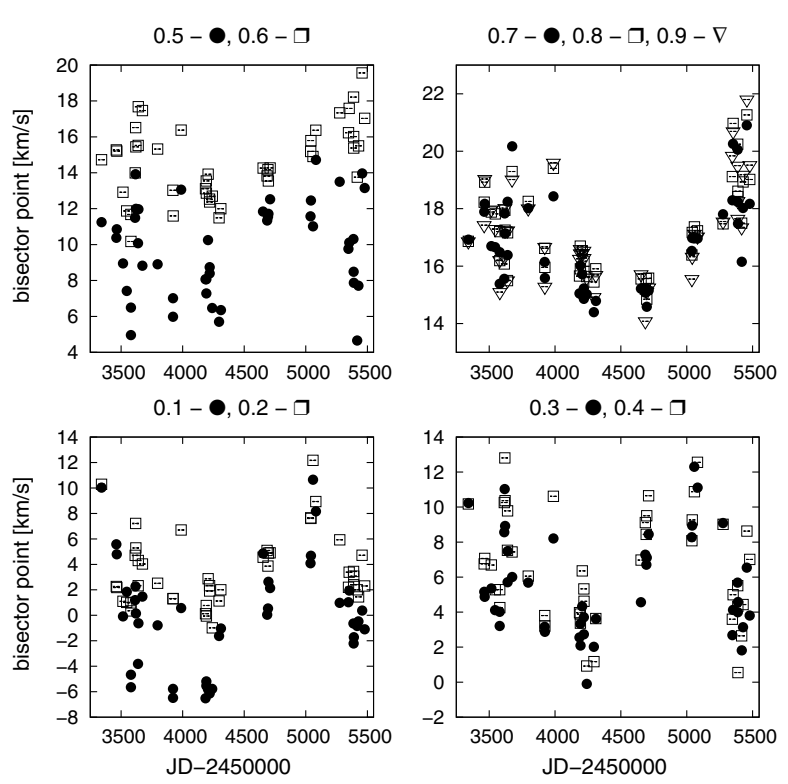

Fig. 7. Bisector points of the $\mathrm{H} \alpha$ line in relative heights 0.1 (wings) to 0.9 (peak).

Three parts of the $\mathrm{H} \alpha$ line can be resolved (Fig. 7). The wings and the lower part of the core (0.1-0.4 of the relative height) appear to have similar kinematical behaviours and in spite of the scatter, the curve with both its minimum and maximum is discernible in the plot. The minimum occurs on JD $2454130 \pm 40$. The relative heights at $0.7-0.9$ represent the peak. The bisectors show that its radial velocity also slightly changes, but in a different way from the velocity variations of the wings. The minimum was observed on JD $2454540 \pm 30$ here. The bisector velocity at half-maximum of the relative height of the line shows some scatter, but no noticeable trend or period.

\section{Radial velocities}

Since V2028 Cyg is assumed to be a binary, we investigated the radial velocity variations in its spectra. The absorption lines of the cool component as well as the emission from the envelope were measured.

The $\mathrm{K}$ type absorption lines are often blended and affected by noise in the Ondřejov spectra. We therefore used the cross-correlation method for our measurements. The ELODIE spectrum reduced to the Ondřejov resolution serves as a template. Nine intervals without emission lines and atmospheric absorption lines were chosen for the measurements (6334-6344.5 $\mathrm{A}, 6398-6415 \AA$, 6434-6440.5 , 6444-6453 $\mathrm{A}, 6607-6626 \AA, 6636-6664.5 \AA, 6680.5-6693 \AA$, $6694.5-6706.5 \AA, 6709-6720 \AA)$. The resulting radial velocities in Fig. 8 are mean values of these intervals and the errors are computed as the standard deviation in the arithmetic mean. The measured mean radial velocities vary slightly, but no periodical variations were found. To check the validity of the results we chose seven unblended absorption lines to measure radial velocities using other methods (Fe I $6355 \AA$, Ca I $6439 \AA$, Cr I $6630 \AA$, Fe г $6647 \AA$, Fe г $6648 \AA$, Fe I $6704 \AA$, Li I $6708 \AA$ ). The shifts in these lines were measured (Fig. 8) using a) Gaussian fitting and b) profile mirroring. The profile mirroring was done using both automatic and manual procedures. The results from both techniques agreed to within 

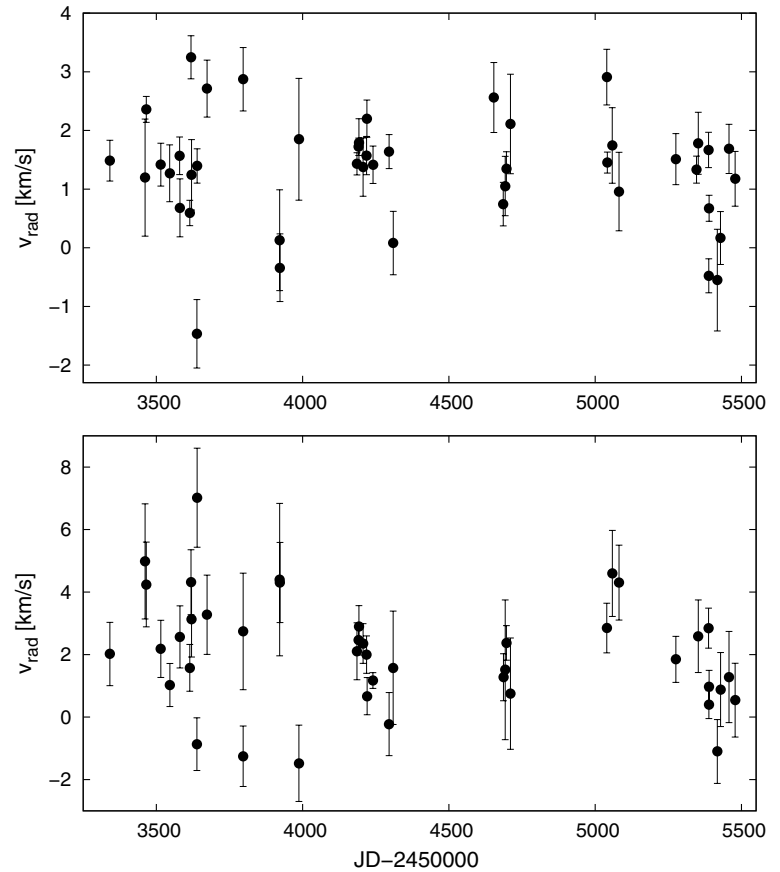

Fig. 8. Radial velocities of the $\mathrm{K}$ type absorption lines measured by cross-correlation (upper panel) and profile mirroring (lower panel).
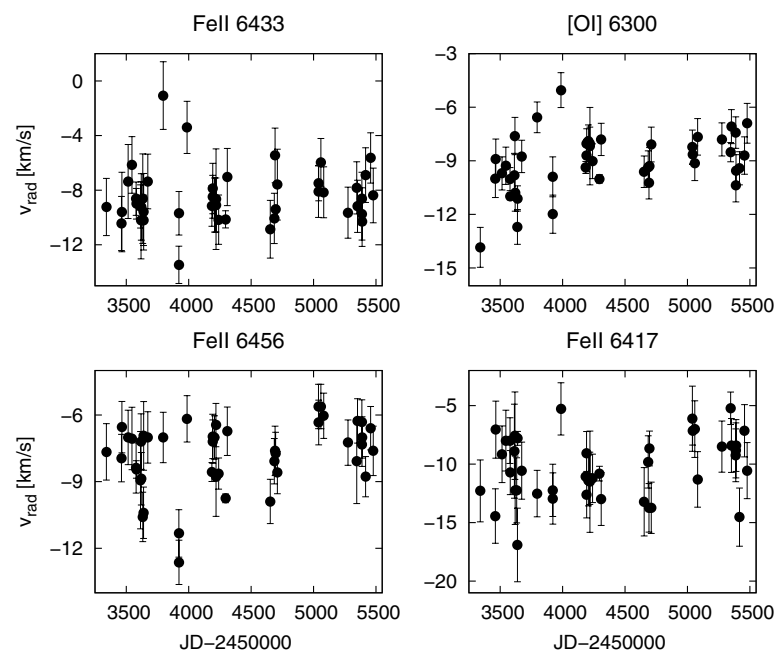

Fig. 9. Radial velocities of metallic emission lines.

the error intervals. The lower panel of Fig. 8 shows the values obtained manually using a program $\mathrm{SPEFO}^{2}$ (developed by Horn, description in Škoda 1996). The scatter and error intervals are approximately double those of the cross-correlation results, although the variations are very similar. There was a decline in the past 200 days and had been an indistinct maximum before it (Fig. 8).

The radial velocities of metallic emission lines (Fig. 9) were derived by Gaussian fitting. There is also some scatter, but this is primarily caused by the noise. No period can also be seen here.

The radial velocities of the $\mathrm{H} \alpha$ line were measured for the peak as well as for the line wings. A polynomial fitting was used to measure the peak velocities. We adopted the method of Mikulášek et al. (2003) based on the least squares method. The Ondřejov spectrograph resolution provides typically six

${ }^{2}$ http://astro.troja.mff.cuni.cz/ftp/hec/SPEF0.263/
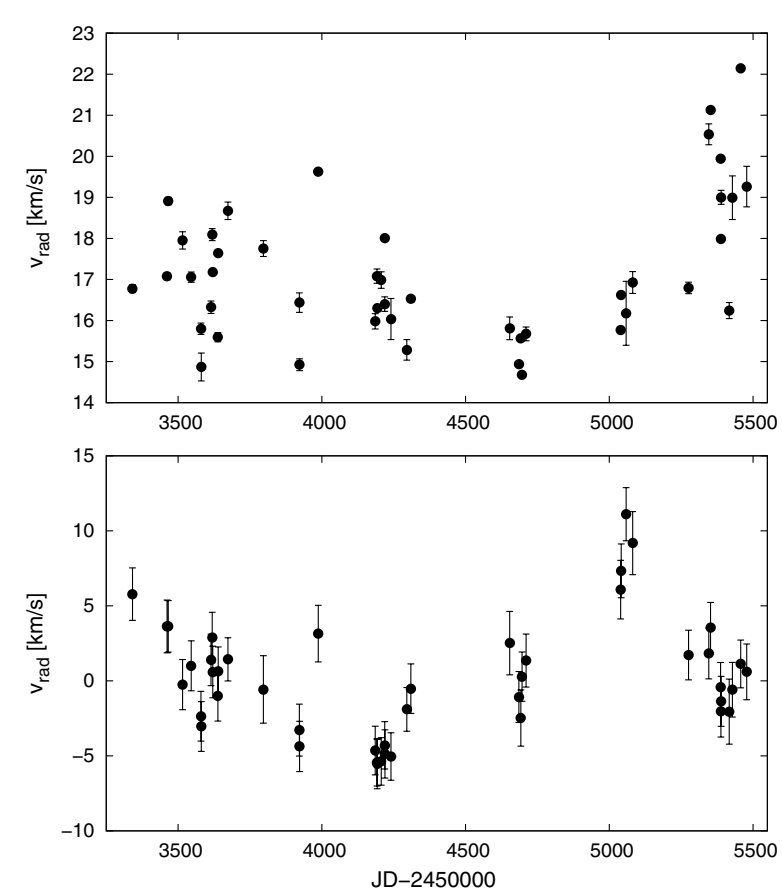

Fig. 10. Radial velocities of the $\mathrm{H} \alpha$ peak (upper panel) and wings (lower panel).

to eight points in the peak interval, which is sufficient to obtain a good fit.

We used a double-Gaussian method (Shafter 1983) to measure the radial velocities of the wings. The method consists of the determination of the line centre $\lambda$ by solving the equation

$\int_{-\infty}^{\infty} S(\Lambda) K(\lambda-\Lambda) \mathrm{d} \Lambda=0$

where

$K(x)=\exp \left[-(x-a)^{2} / 2 \sigma^{2}\right]-\exp \left[-(x+a)^{2} / 2 \sigma^{2}\right]$

and $S(\Lambda)$ is the investigated spectrum. The errors were computed according to the relation by Horne et al. (1986). The parameter $a$ adjusts the separation of the Gaussians and should be chosen according to the width of the wings and the core. Since the Gaussian parameter $\sigma$ is limited by the spectral resolution, the lowest possible value in our case is $0.25 \AA$. However, a larger $\sigma$ value enables us to measure the wider wavelength interval of the wings and it deals better with noise. We adopted values $a=5.0 \AA$ and $\sigma=1.0 \AA$. The credibility of this method was verified by comparing with the results obtained by the profile mirroring method.

Figure 10 (lower panel) shows the minimum and maximum of the radial velocities of the wings. The position of the minimum is JD $2454155 \pm 17$. The radial velocities of the $\mathrm{H} \alpha$ peak are plotted in the same figure (upper panel). The results are different from those for the wing velocities. There is a large scatter in the data but it is possible to resolve the minimum at JD $2454683 \pm 33$.

\section{Discussion and conclusions}

We have measured the equivalent widths and radial velocities of several emission and absorption lines in the spectra 
of V2028 Cyg. We have also thoroughly investigated the profile variations in the $\mathrm{H} \alpha$ line.

The emission lines of metals (the most common of which is Fe II) have a characteristic bluewards skewed profile, which is indicative of an outflowing envelope. The line profile width at half-maximum is usually $\sim 45-55 \mathrm{~km} \mathrm{~s}^{-1}$. The stronger lines (two times the continuum intensity) possess wings of widths up to $\sim 200 \mathrm{~km} \mathrm{~s}^{-1}$. Assuming spherical symmetry, this line profile is indicative of a slow (up to $100 \mathrm{~km} \mathrm{~s}^{-1}$ ) outflow with a small velocity dispersion. Equivalent widths and radial velocities of the metallic emissions display some scatter but no periodic variations. The slight variations in the results may be ascribed to fluctuations in the envelope.

The forbidden lines are in the chosen interval $(\lambda=$ 6250-6770 A) represented mainly by [O I] emission. In addition, their profiles are skewed bluewards but, however, lack the broader wings. They therefore probably originate in the outer, less dense layers of the envelope.

The radial velocities of the $\mathrm{K}$ component measured for the single, most clearly defined absorption lines (derived using both the mirroring method and by fitting the Gaussian profile) have a similar behaviour to the $\mathrm{H} \alpha$ wings. However, the cross-correlation method, even if the chosen intervals are unaffected by water absorption, gives less significant results. Since our spectra are affected by a large amount of noise, the radial velocities obtained from the measurements of the most clearly defined spectral lines are probably more reliable than the latter. Furthermore, accurate observations are necessary to (dis)confirm the correlation of the radial velocities of the $\mathrm{H} \alpha$ wings with those of the absorption lines. This relation can be important when determining the role of binarity in this system. The radial velocity variations of the absorption component in our data are relatively small $\left(<8 \mathrm{~km} \mathrm{~s}^{-1}\right)$ and fall into the same interval as the measurements of Zickgraf (2001). This implies a long orbital period for V2028 Cyg with a lower limit of 25 years.

The Balmer lines $\mathrm{H} \alpha, \beta$, and $\gamma$ are the strongest lines in the spectrum (approximately an order of magnitude stronger than the metallic emissions). All of them are asymmetric with the peak shifted redwards with respect to the wings. In most spectra, the lines bear a hump on the blue side, approximately at the height of the half-maximum. The $\mathrm{H} \delta$ emission is also clearly visible but any specific features of the profile are overlaid by the noise. From this set, only $\mathrm{H} \alpha$ has a sufficient coverage in the six-year-long data set from Ondřejov used to study its variations. It is also a strongest emission line in the spectrum. For this line, we measured the variations with time in the equivalent width, intensity, radial velocity, and bisector values.

Our observations uncover some of the general properties of the line profiles and correlations between measured quantities:

- H $\alpha$ line

- The line profile is observed in nearly all spectra to be asymmetric. All of these observations show a red shift of the line peak relative to the centre defined by the wings. The symmetric line profile is very rare in our data.

- The radial velocities and bisectors vary differently in the wings and peak.

- The absolute value of the equivalent width $|E W|$ is correlated with the maximum flux I (Fig. 3).
- The absolute value of $|E W|$ is anti-correlated with the radial velocity of the wings $v_{\text {rw }}$ (Figs. 3 and 10).

- The equivalent width of [OI] $6300 \AA$ A, Fe II 6427, 6433, and $6456 \AA$ lines do not show any significant changes.

- The radial velocities of the $\mathrm{K}$ absorption component may correlate with the $\mathrm{H} \alpha$ wings. However, further observations of higher resolution are necessary to confirm this property.

These results can be compared with the results of future modelling. In particular, the anticorrelation of the absolute value of the $\mathrm{H} \alpha$ equivalent width and the radial velocities of its wings probably strongly restricts on the proposed model.

V2028 Cyg is a very complicated system to model. It is necessary to take into account the binary nature of the object. A particularly large problem is the extended circumstellar region, which probably forms a disc-like structure. These properties require a combination of at least 2D time-dependent hydrodynamic and NLTE radiative transfer in moving media. Although our observations have not revealed the real nature of V2028 Cyg, they specify the model properties for future investigation. Particular model properties can be tested using our observations.

Acknowledgements. We thank especially Anatoly Miroshnichenko for his valuable and inspiring advice and remarks. We also thank P. Hadrava, P. Koubský, and P. Harmanec for their advice and Adela Kawka for taking some of the spectra in her observation time. This research is supported by grants 205/09/P476 (GA ČR), 205/08/H005 (GA ČR), MUNI/A/0968/2009, and MSM0021620860 (MŠMT ČR). The Astronomical Institute Ondřejov is supported by a project AV0Z10030501.

\section{References}

Allen, D. A. 1973, MSRSL, 5, 335

Allen, D. A. 1974, MNRAS, 168, 1

Allen, D. A., \& Swings, J. P. 1976, A\&A, 47, 293

Arkhipova, V. P., \& Ipatov, A. P. 1982, SvAL, 8, 298

Bergner, Yu. K., Miroshnichenko, A. S., Yudin, R. V., et al. 1995, A\&AS, 112, 221

Castor, J. I., Abbott, D. C., \& Klein, R. I. 1975, ApJ, 195, 157

Corporon, P., \& Lagrange, A.-M. 1999, A\&AS, 136, 429

Horne, K., Wade, R. A., \& Szkody, P. 1986, MNRAS, 219, 791

Korčáková, D., \& Kubát, J. 2005, A\&A, 440, 715

Korčáková, D., Nagel, T., Werner, K., Suleimanov, V., \& Votruba, V. 2011, A\&A, 529, A119

Lamers, H. J. G. L. M., Zickgraf, F.-J., de Winter, D., Houziaux, L., \& Zorec, J. 1998, A\&A, 340, 117

Marston, A. P., \& McCollum, B. 2008, A\&A, 477, 193

Merrill, P. W., Burwell, C. G., \& Miller, W. C. 1942, ApJ, 96, 15

Mikulášek, Z., Žižňovský, J., Zverko, J., \& Polosukhina, N. S. 2003, Contrib. Astron. Obs. Skalnaté Pleso, 33, 29

Miroshnichenko, A. S. 2007, ApJ, 667, 497

Miroshnichenko, A. S., Chentsov, E. L., \& Klochkova, V. G., et al. 2000, A\&AS, 147,5

Moultaka, L., Ilovaisky, S. A., Prugniel, P., \& Soubiran, C. 2004, PASP, 116, 693 Pych, W. 2004, PASP, 116, 148

Shafter, A. W. 1983, ApJ, 267, 222

Škoda, P. 1996, ASPC, 101, 187

Škoda, P., Šlechta, M., \& Honsa, J. 2002, AICz, 90, 3

Steffen, M. 1990, A\&A, 239, 443

Vollmann, K., \& Eversberg, T. 2006, AN, 9, 865

Votruba, V., Feldmeier, A., Kubát, J., \& Rätzel, D. 2007, A\&A, 474, 549

Zickgraf, F.-J., 2001, A\&A, 375, 122

Zickgraf, F.-J., \& Schulte-Ladbeck, R. E. 1989, A\&A, 214, 274

Zickgraf, F.-J., \& Stahl, O. 1989, A\&A, 223, 165

Zickgraf, F.-J., Wolf, B., Stahl, O., Leitherer, C., \& Klare, G. 1985, A\&A, 143, 421 